\title{
Post Mortem Pink Teeth and Pseudo Pink Nails in a Case of Suicidal Hanging
}

\author{
Pawan Mittal \\ Demonstrator, Department of Forensic Medicine, Bhagat Phool Singh Medical College for Women, Sonipat, India \\ Email address: \\ drmittalpawan@gmail.com

\section{To cite this article:} \\ Pawan Mittal. Post Mortem Pink Teeth and Pseudo Pink Nails in a Case of Suicidal Hanging. Pathology and Laboratory Medicine. \\ Vol. 5, No. 1, 2021, pp. 1-5. doi: 10.11648/j.plm.20210501.11
}

Received: January 4, 2021; Accepted: January 12, 2021; Published: January 22, 2021

\begin{abstract}
A postmortem case of suicide by hanging is described where pink teeth and pink nails were found during autopsy. One highly putrefied dead body belonging to a middle aged man was found hanging from the branch of a tree amidst a forest. Multiple permanent teeth and bilateral little fingernails displayed pink discolorations. After considering the circumstances of the case and autopsy findings including the ambiguity over pink tissue phenomenon, the cause of death was finally given as ante mortem hanging. The pink teeth phenomenon [PTP] is an infrequent and dubiously explained finding in forensic literature while a single case report exists in relation to pink nails. Although many authors have stressed that post-mortem pink teeth should not be considered as a reliable dental parameter for determining the cause of death, the results of other studies have shown that the pink teeth commonly occur in cases of asphyxia such as strangulation, drowning or suffocation. The finding, although initially related to an intravitam cranial congestion, was subsequently refuted on account of its discovery in nonasphyxial and other modes of deaths. A potential role of postmortem hypostatic congestion in this regard has been proposed especially in drowned corpses due to their frequent floatation in a head down position. A moist and humid environmental milieu along with certain degree of putrefaction has been considered as a prerequisite for the enhancement of the process. The repeated discovery of the finding in autopsy cases has been almost a continuing conundrum for the pathologists. The pink tissue phenomenon mandates a sound and goal oriented investigation in order to clarify its role in ascertaining the cause of death.
\end{abstract}

Keywords: Pink Teeth, Pink Nail, Dentine, Asphyxia, Hemoglobin, Congestion

\section{Introduction}

Permanent or transient pigmentation of the teeth can occur due to extrinsic or intrinsic causes [1]. The changes can be generalized or localized [2]. The term 'pink tooth' refers to a pink to red coloration of the dentine, which becomes particularly deep towards the cement enamel junction and fading off towards both ends [2]. The first description of pink teeth phenomenon [PTP] was made by Thomas Bell in 1829 [1]. However, the path gnomic value of pink teeth has been more than doubtful since it has been described in a variety of unnatural and violent deaths such as asphyxiation, blunt, sharp and gunshot trauma, poisonings and intoxications [2-7], as well as with unknown/unsolved cause and manner of deaths [8] and archeological situations [9].

Similar discoloration has been also described in a case involving multiple fingernails in a mummified body [10].
We hereby report the phenomenon of postmortem pink teeth and an additional finding simulating pink nails in a middle aged man, who committed suicide by hanging.

\section{Case Details}

\subsection{History}

The case belongs to a 45 years old married male, shopkeeper by profession, with a long history of depression since his mother's demise. Any history of medication or physical illness was absent. About 6 days before discovery of his dead body, he had left home as a routine but did not come back, unless one day a stranger passing through a distant forest noticed a foul smelling putrefied body hanging from a tree and informed the local police. The police reached the spot and conducted the inquest on the grounds of an 
unknown body committing suicide by hanging. Identification and history of the deceased were established 2 days after its discovery, by means of a newspaper details.

\subsection{Crime Scene Findings}

The scene was descriptive of an apparently decomposed male body, hanging from the branch of a tree amidst a forest [Figure 1]. The ligature consisted of a pink cotton male parna [scarf] that was carrying an atypical knot over the left mastoid-occipital region thereby causing a slight neck tilt to the right and downwards. Toes of the right foot were just touching the ground while the left foot was dangling free in the air. No part of the body was in contact with the tree. The deceased was wearing a multicolored checked shirt and a grayish formal pant which were overstretched while a golden watch was visible around left wrist. The feet were bare. Scalp hairs were overtly smudged with eggs of flies, primarily over the occiput. Flies were settled over the clothes while few maggots could be seen over the face. Facial skin was seemingly parched, dark reddish to blackish, suggestive of an incipient or overt mummification. Identification was difficult from facial features alone, although a tattoo mark on the middle front of left forearm turned out to be of immense help

As per police information, the surroundings were shaded, warm, and slightly humid having free access of air. The body was visible through long standing bamboo twigs and plants. The four limbs were flexed and abducted apart, due to gaseous distension.

Overall the scene had an impression of a classical hanging. A suicide note was recovered from back pocket of his pant, clearly stating his reasons for giving up life. The spouse confirmed of the personal possession of ligature by the deceased.

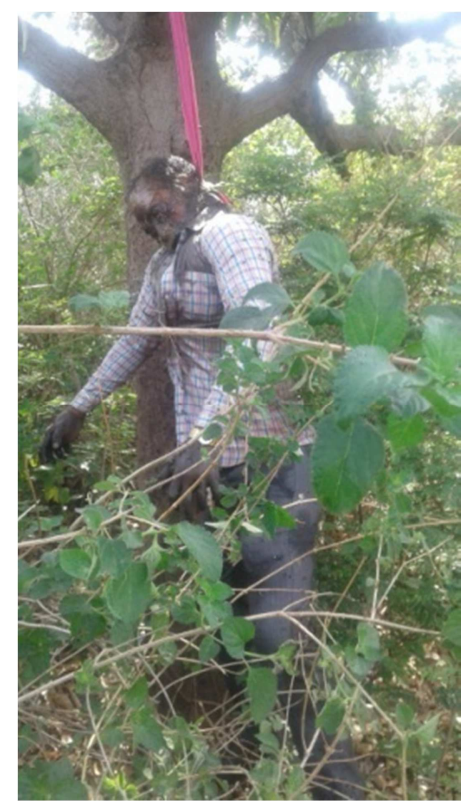

Figure 1. Crime scene image showing dead body hanging from a tree with an atypical knot over left side of the neck along with a right and down tilt. Putrefaction is established. Facial skin appears to be mummified.

\subsection{Autopsy Details}

The dead body was brought for postmortem examination on next day. A pale brownish ligature mark was present around the neck, on the background of a darkened and peeled off epidermis, taking the course and pattern of ligature that was already visible at the scene. It carried a fixed atypical knot over left mastoid region and was loose around the neck. The neck was slightly compressed. The mouth was wide open. The eyeballs were distorted, black and parched. Hyoid bone and laryngeal cartilages were ossified and intact, as well as the tracheal rings. Carotids and jugulars vessels lacked any ecchymoses or intimal tears. Cervical vertebrae were intact. Soft tissues and muscles of neck were free of any blood extravasations. The facial skin above ligature was darkened along with changes of partial mummification. Facial features were identifiable with difficulty. Oral cavity was full of maggots while patches of fungus along with clusters of eggs of flies were randomly distributed around head and neck. Heavy maggot infestation had created sieve like areas all over the body. Degloving of the hands and feet was present.

Slight hemolytic congestion over clavicular periosteal surface of left stern mastoid muscle's origin was present. Ventral aspects of intervertebral discs between L1 to L4 lumbar vertebrae depicted dark reddish hemolytic stripes, reminiscent of Simon's bleeding, while the finding was missing on upper as well as lower discs [Figure 2].

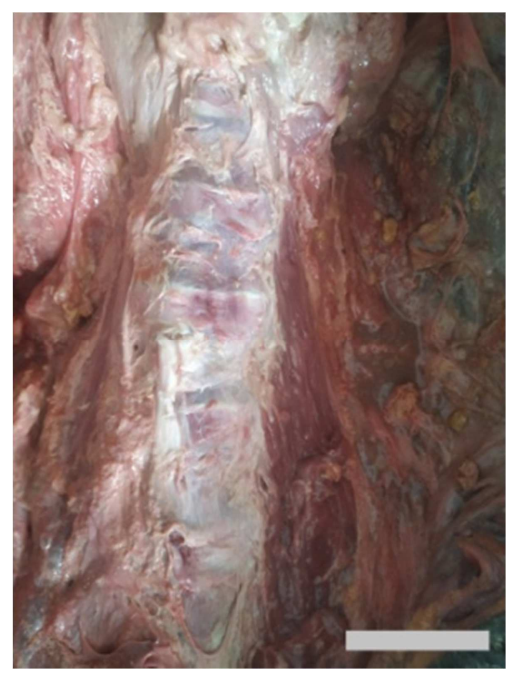

Figure 2. Dark red hemolytic staining of lumbar intervertebral discs [reminiscent of Simon's bleed]; absent in the discs situated above and below.

No additional body injury was present. On the basis of circumstantial facts and autopsy findings, the cause of death was given as ante mortem hanging. Toxicology results were negative.

Upon dental examination, multiple maxillary and mandibular teeth, especially anterior and majority of the right posterior, depicted pink tooth phenomenon [Figure 3]. The discoloration was more intense over neck and root regions while fading towards on either ends. The available teeth were loosened in the sockets and showed considerable attrition over occlusal surface with exposed dentine. 


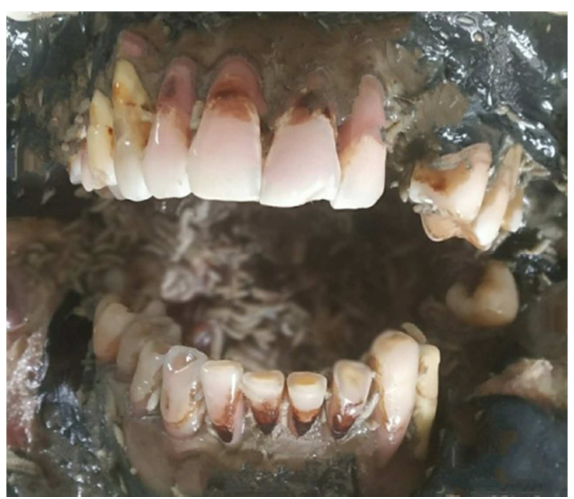

Figure 3. Multiple pink teeth, especially involving anterior and right rows. Note the non-pink right maxillary canine and first premolar in between pink teeth.

A dental chart including details of pink teeth has been prepared [Figure 4].

Additionally, a superficial pink discoloration involving nails of bilateral little fingers was found. The finding initially raised the suspicion of a true pink nail phenomenon, but on closer scrutiny the surfaces were seemingly rough and gritty on touch while the discoloration was found to be located superficially [Figure 5]. Furthermore, the colour was easily scratched and cleaned off by means of a scalpel and absolute alcohol, thereby revealing it to be the nail paint/varnish. The underlying blackish nail bed became clearly visible that was further confirmed by nail removal. Therebythe pink staining of both nails proved to be a simulation rather than a medicolegally relevant finding.

A postmortem interval of 7 to 10 days was opined on the basis of the history and postmortem changes.

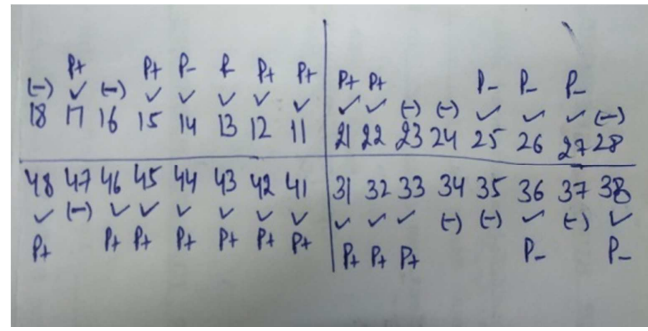

Figure 4. Dental chart along with details of pink teeth.

Key:

$\checkmark$ : Tooth Present; [-]: Tooth missing with absorbed socket P+: PTP present; P-: PTP absent [PTP: Pink Teeth Phenomenon]

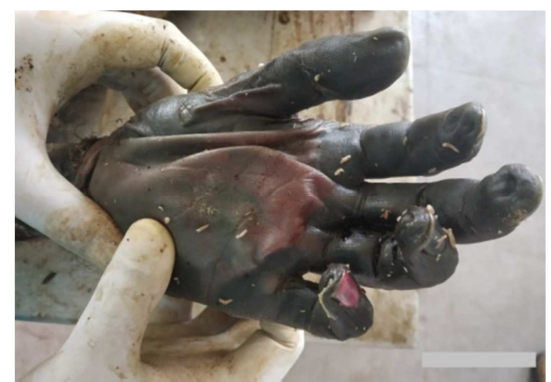

Figure 5. Pink nail paint applied over left little finger [right fingernail was also polished], simulating pink nail phenomenon.

\section{Discussion}

Although the phenomenon of pink teeth has been known since 1829, when it was first described by Bell, its application in forensic medicine has been limited [1, 11]. There are several theories to explain the phenomenon of pink teeth and all of them suggest that the pink color is induced by the presence of hemoglobin inside the pulp chamber, due to the accumulation of blood in the cephalic segment $[2,12]$.

The appearance of pink staining in isolated tooth has been known in living cases of typhoid fever, porphyria and postendodontic procedures [13-15]. The coloration has been predominantly found in postmortem cases of asphyxial and other congestive modes of deaths that cause increased cephalic venous pressure leading to rupture of vessels and leakage of blood in the pulp chamber $[16,17]$.

The occurrence and the long time stability of the pink colour has been attributed to special anatomical conditions of dentin such as the existence of porous structures in the form of dentinal tubules in the hard and dense dentin [10]. The leakage of hemoglobin or derivatives into the dentinal tubules associated with autolysis, temperature, humidity and the position in which the bodies were found, are considered to assist in the development of pink teeth $[2,18]$. Therefore, they are most often found in drowning asphyxiation $[12,18]$. Mechanical asphyxia by strangulation and hanging significantly increase the incidence of pink tooth formation, due to blood stagnation in the cephalic segment $[7,11,12$, 19].

Due to discovery of pink teeth in autopsy cases of deaths from bleeding gunshot, blunt and sharp injuries as well as intoxications, poisonings, negative autopsy and archeological situations, it has been repeatedly declared as a non-specific phenomenon $[6,8,9,11,12,18,20]$.

A large amount of blood on account of congestion or hemorrhage must be present in the pulp chamber in order to release enough hemoglobin derivatives to penetrate the hard tissues of the teeth [20]. The phenomenon has been explained through in vitro studies by using cadaver blood and sectioned tooth [21] and also by instilling blood and hemolysed erythrocytes into the pulp chambers [22]. The finding may involve from a single to virtually every tooth in the either or both jaws [22, 23]. The pigmentation is more prominent in the anterior teeth with single roots than the posterior teeth with multiple roots $[24,25]$. Teeth with cavities are less pink than healthy teeth, due to an initial reduction in pulp volume and subsequent reduced amount of blood in the pulp chamber [5]. Furthermore completely unstained teeth have been discovered in between pink teeth $[4,5,20]$, even with anterior teeth unaffected [4]. In our case, right maxillary canine and first premolar were free of any pink discoloration.

The preferential staining of a few teeth over the other has been suggested on the basis of dependent position of the head [20]. The role of a hypostatic phenomenon, although, has been doubted in a study showing pink discoloration in the non dependent/contra lateral teeth [4]. In our case, although to exactly delineate an intravitam congestion [due to venous 
occlusion] from the hypostatic process was not possible but an absence of left sided [posterior] pink teeth suggest hypostasis to be the predominant process that occurred due to right and downward tilt of the neck. A nearly free hanging position of the body further points towards an ischemic mechanism of death, although it is more of a knot-location dependent phenomenon [26]. In this regard a leading role of degree of putrefaction along with atmospheric moisture and humidity and body position have been proposed as leading factors for the occurrence of pink teeth $[2,16]$.

Ortmann and Du Chesne have described similar pink discolorations in the teeth as well as nails of bilateral thumbs and left little finger in a putrefied and partially mummified female corpse which was found in a forest and died due to combined effect of trimipramin intoxication, hypothermia and pneumonia [10]. The application of nail varnish was ruled out by cleaning them with acetone while the real cause was demonstrated in the nail beds after removal of the nails. Furthermore, the subungual tissue did not reveal any $\mathrm{CO}-\mathrm{Hb}$ by gas chromatography [10]. Our case revealed simulation of the pink nail phenomenon, an indication furthermore, to anticipate utmost care while dealing with such cases.

The pink teeth finding has a biological predilection for young ages where dentinal tubules have a larger diameter in comparison to older teeth [5]. Another factor is a constant reduction in the volume of the pulp and pulp chamber space due to the deposition of secondary dentin as the person ages [27].

The increased fibrinolytic activity in the pulp chamber in situations of sudden, unnatural and violent deaths facilitates the breakdown of coagulated blood or prevents postmortem coagulation in the pulp chamber and with the hemolysis of erythrocytes it allows the diffusion of hemoglobin in the dentinal tubules $[11,28]$. This fact has been further related to hypoxia induced hypercatecholanemia [28]. However, the studies involving large case material have not reported pink teeth in natural deaths $[8,18]$. In this regard, it has been stated that people who die a natural death are not usually placed with their head in a prone position to allow the blood to drain into the teeth [20].

With methods such as immunohistochemistry and autoflourescence, the causal pigment has been identified as undegraded hemoglobin $[10,12]$. Involvement of hemosiderin, bile pigments, related bile and porphyrins was discarded [5, 12]. However, the exact etiology behind pink coloration taken on by the hemoglobin [seemingly under pressure] within the pulp cavity is less clear [8].

The color of the pink teeth can only occur after hemolysis, and the discoloration has been shown to become evident macroscopically in about 6 days and peaks at about 18 days [29]. However different studies have suggested different time periods so the time of occurrence of PTP has been considerably variable $[5,17,23]$. Furthermore it has to be understood that the time of analysis and time of development of pink teeth may not be the same [16]. This fact carried important legal bearing.

Pink teeth have been found in the dead bodies that had been recovered after prolonged post mortem interval, such as putrefied corpses or cases depicting various modifications of decomposition and their formation is a time dependent phenomenon.

The non-specificity and the long time stability of the pink discoloration suggests that pink teeth are not completely uncommon in putrefied corpses.

\section{Conclusion}

In our case, changes of putrefaction in the head, right and dependent neck position favoring hypostatic accumulation of blood, presence of warm and humid surroundings and a dubiously involved congestive process; all together must have contributed to the formation of pink teeth. The pink fingernails finally turned out to be artificially applied nail paint; it shall be appropriate to label them as "Pseudo Pink Nails". A cursory and heedless observation may result in mislabeling such artefacts as true pink phenomenon which may result in legal jeopardize. The postmortem pink teeth and pink nail phenomenon must be studied in order to understand their importance in medico-legal context, as up to now their exact relationship with the cause of death is not known. Till then we maintain that these are non-specific findings and must not be misinterpreted in medico-legal investigations.

\section{Conflicts of Interest}

The author does not have any possible conflicts of interest.

\section{References}

[1] Bell T. The Anatomy, Physiology and Diseases ofthe Teeth. $2^{\text {nd }}$ Edition. London: Stuart and Co; 1835.

[2] Van Wyk CW. Pink teeth of the dead: 1. A clinical and histological description. J Forensic Odontostomatol. 1987; 5 (2): 41-50.

[3] Furuhata T, Yamamoto K. Forensic odontology. Thomas: Springfield; 1976.

[4] Clark DA, Law ML. Post-mortem pink teeth. Med Sci Law. 1984; 24: $130-4$.

[5] Kirkham WR, Andrews EE, Snow CC et al. Postmortem pink teeth. J Forensic Sci. 1977; 22: 119-31.

[6] Franco A, Mendes SDSC, Picoli FF, Rodrigues LG, Silva RF. Forensic thanatology and the pink tooth phenomenon: From the lack of relation with the cause of death to a potential evidence of cadaveric decomposition in dental autopsies Case series. Forensic Sci Int. 2018 Oct; 291: e8-12.

[7] Brites AN, Rezende Machado AL, Franco A, Alves da Silva RH. Revisiting autopsies of death by mechanical asphyxia in the search for post-mortem pink teeth. J Forensic Odontostomatol. 2020 May 15; 1 (38): 34-8.

[8] Beeley JA, Harvey W. Pink teeth appearing as a postmortem phenomenon. J Forensic Sci Soc. 1973; 13: 297-305. 
[9] Dye TJ, Lucy D, Pollard AM. The occurrence and implications of post-mortem 'pink teeth' in forensic and archaeological cases. Int J Osteoarchaeol. 1995; 4: 339-48.

[10] Ortmann C, Du Chesne A. A partially mummified corpse with pink teeth and pink nails. Int J Legal Med. 1998; 111 (1): 35-7.

[11] Montenegro JB, Queiroz EGAL, Moreno BLG, De Souza EHA, De Carvalho, Soriano EP. Dentes Rosado observado quarto mesesapos a morte. Der Cambio Soc. 2013; 1: 1-7.

[12] Campobasso CP, Di Vella G, De Donno A, Santoro V, Favia $\mathrm{G}$, Introna F. Pink teeth in a series of bodies recovered from a single shipwreck. Am J Forensic Med Pathol. 2006; 27 (4): 313-6.

[13] Oguz F, Sidal M, Bayram C, Sansoy N, Hekim N. Ocular involvement in two symptomatic congenital erythropietic porphyria. Eur J Pediatr. 1993; 152 (8): 671-3.

[14] Fuss Z, Tsesis I, Lin S. Root resorption-diagnosis, classification and treatment choices based on stimulation factors. Dent Traumatol. 2003; 19 (4): 175-82.

[15] Bergenholz G. Iatrogenic injury to the pulp in dental procedures: aspects of pathogenesis, management and preventive measures. Int Dent J. 1991; 41 (2): 99-110.

[16] Borrman H, Du Chesne A, Brinkmann B. Medico-legal aspects of postmortem pink teeth. Int J Legal Med. 1994; 106 (5): 225-31.

[17] Sainio P, SyrjXnen S, Kellgla J, Parvianen A. Postmortem pink teeth phenomenon: an experimental study and a survey of the literature. Proc Finn Dent Soc. 1990; 86: 29-35.

[18] BrondumN, Simonsen J. Postmortem red coloration of teeth: a retrospective investigation of 26 cases. Am J Forensic Med Pathol. 1987; 8 (2): 127-30.

[19] Soriano EP, de Carvalho MVD, Santos FBD, de Mendoza CC, de AraújoMdSD, Campello RIC. The post-mortem pink teeth phenomenon: a case report. Med Oral Patol Oral Cir Bucal. 2009 Jul 1; 14 (7): e337-9.

[20] Van Wyk CW. Pink teeth of the dead: II. Minor Variations. J Forensic Odontostomatol. 1988a; 6: 35-42.

[21] Van Wyk CW. Postmortem pink teeth: in vitro production. J Oral Pathol Med. 1988b; 17: 568-72.

[22] de Almeida CA, Daruge E, Daruge E Jr, el-Guindy M. Comparative study of experimentally induced and postmortem pink teeth. J Forensic Odontostomatol. 1996; 14 (2): $25-7$.

[23] Whittaker DK, Thomas VC, Thomas RIM. Post-mortem pigmentation of the teeth. Br Dent J. 1976; 140: 100-2.

[24] Miller SC. Oral Diagnosis and Treatment. New York: Blackstone; 1957.

[25] Franco A, de Oliveira MN, Gomes-Lima LK, Pereira-deOliveira VHF, Franco RPAV, Bluenberg C, et al. Case-specific characteristics of pink teeth in dental autopsies - A systematic review. J Forensic Leg Med. 2019; 68: 101869.

[26] Keil W. Obstruction of the Respiratory Orifices, Larynx, Trachea and Bronchia. In: Madea B, editor. Asphyxiation, Suffocation and Neck pressure Deaths. $1^{\text {st }}$ Edition]. CRC Press, Taylor \& Francis Group: Boca Raton, London, New York; 2021.

[27] Sakuma A, Saitoh H, Ishii N, Iwase H. The effects of racemization rate for age estimation of pink teeth. J Forensic Sci. 2015; 60 (2): 450-2.

[28] Berg S. Grundriss der Rechtsmedizin. 12 ${ }^{\text {th }}$ Edition. Muller Steinicke: Munchen; 1984.

[29] Van Wyk CW. Postmortem pink teeth. Histochemical identification of the causative pigment. Am J Forensic Med Pathol. 1989; 10: 134-9. 